\title{
Alignment of Confocal Scanning Laser Ophthalmoscopy Photoreceptor Images at Different Polarizations Using Complex Phase Relationships
}

\author{
Alexander Wong, Member, IEEE
}

\begin{abstract}
A polarimetric technique for enhancing fundus images was recently introduced [8], where confocal scanning laser ophthalmoscopy (CSLO) images are acquired under differing incoming polarization states, and spatially resolved Mueller images are constructed based on the images. An important stage in this technique is the alignment of CSLO images acquired under differing polarization states. This has proven to be particularly difficult when dealing with photoreceptor images, which are characterized by poor SNRs and intensity inhomogeneities due to polarization properties. In this paper, an automated approach to aligning CSLO photoreceptor images acquired under differing polarization states is presented. A novel energy functional based on complex phase relationships is introduced that is invariant to polarization and scale, as well as robust to noise and highly sensitive to photoreceptor structural characteristics. A sequential quadratic programming approach is employed to determine the optimal alignment between the photoreceptor images by minimizing the proposed energy functional. The method has been tested on CSLO fish photoreceptor images acquired under differing polarization states and evaluated based on alignment accuracy. The results demonstrate that the proposed method outperforms existing techniques used for aligning CSLO images, with lower mean alignment error for all test cases.
\end{abstract}

Index Terms-Alignment, complex phase, ophthalmoscopy, photoreceptor, polarization.

\section{INTRODUCTION}

$\mathbf{R}$ ETINAL dystrophies refers to a group of incurable eye diseases that result in progressive deterioration of the retina. Common retinal dystrophies include cone-rod dystrophies (CRDs) and retinitis pigmentosa (RP), which are characterized by the gradual degradation of rod and cone photoreceptors. In CRDs, patients typically exhibit a loss of visual acuity, color vision deficiencies, as well as loss in central vision sensitivity in the early stages followed by the loss of peripheral and night vision. This sequence of events is due to the fact that CRDs are typically characterized by a primary loss in cone photoreceptors and a secondary loss in rod photoreceptors [1], [2]. In RP, patients typically experience loss of peripheral and night vision in the early stages, followed by loss in color vision and visual

Manuscript received September 19, 2008; revised December 17, 2008. First published March 27, 2009; current version published June 12, 2009. This work was supported in part by the Natural Sciences and Engineering Research Council of Canada.

The author is with the Systems Design Engineering, University of Waterloo, Waterloo, ON N2L 3G1, Canada (e-mail: a28wong@engmail.uwaterloo.ca).

Color versions of one or more of the figures in this paper are available online at http://ieeexplore.iee.org.

Digital Object Identifier 10.1109/TBME.2009.2017510 acuity in later stages due to the primary loss of rod photoreceptors followed by a secondary loss of cone photoreceptors [3]. In particular, RP represents the most prevalent cause of vision loss among the working population in developed countries [3]. Given the debilitating effects of retinal dystrophies, it is necessary to systematically study changes in photoreceptors to get a better understanding of the underlying mechanisms of photoreceptor abnormalities, as well as improve clinical diagnosis and quantification of retinal disease severity.

Traditionally, most of the knowledge regarding retinal deterioration and abnormalities come from postmortem studies of the retina. While this has contributed greatly to the understanding of the underlying mechanisms associated with retinal diseases, such studies are of little use for studying retinal changes over time as well as clinical diagnosis and disease severity assessment. More recently, advances in ophthalmoscopic imaging has allowed for in vivo imaging of the retina [2], [4]-[8]. This has given important insights in the relationship between retinal changes and the visual function of patients suffering from different forms of retinal dystrophy. For example, it was observed by Choi et al. [2] that the retina of patients with retinal dystrophy exhibited irregularities in cone photoreceptor densities when compared to healthy retinas. Furthermore, it was found that the extent of such irregularities is highly correlated to the visual function of the patient, thus making this form of analysis useful for assessing disease severity. Of particular interest among the recent ophthalmoscopic imaging techniques is that proposed by Bueno and Campbell [6] and Bueno et al. [8], which utilizes the polarization properties of fundus structures to improve and enhance the visualization of fundus images acquired using confocal scanning laser ophthalmoscopy (CSLO). This enhancement process is very important as the reliability of retinal disease diagnosis is highly dependent on the fundus image quality [8].

One of the key steps in the CLSO fundus image enhancement technique proposed by Bueno et al. [8] is the alignment of CLSO images acquired under different polarization states. This alignment step is particularly necessary in the case of photoreceptor imaging, where even small movements during the in vivo imaging process results in very significant misalignments in the CLSO images. A number of techniques have been proposed for aligning retinal images captured using ophthalmoscopic imaging [5], [8], [9], all based on the concept of using the normalized intensity correlation coefficient (NICC) as an energy functional for obtaining the optimal alignment between the images. However, there are several drawbacks to these methods that may 
hinder their effectiveness for aligning CLSO photoreceptor images acquired under different polarization states. First, CLSO photoreceptor images are characterized by low SNR and poor structural contrast [5]. Both the alignment methods proposed by Goatman et al. [9] and Bueno et al. [8] rely on image averaging of temporally adjacent CLSO images to reduce the effect of noise, while the technique proposed by Wade and Fitzke [5] rely on a large-window averaging filtering process. However, the use of these simple noise suppression techniques may be insufficient in low-SNR situations and may also result in excessive blurring that significantly reduces photoreceptor structural detail. Given the low contrast nature of CLSO photoreceptor images, this oversmoothing of structural detail can be highly problematic and makes it difficult to achieve accurate alignments. Furthermore, due to the complex polarization properties, the CLSO photoreceptor images exhibit intensity inhomogeneities that differ based on the polarization state used during the image acquisition process [8]. While such differing intensity inhomogeneities are exploited to enhance fundus images in the CLSO technique proposed by Bueno et al. [8], they also make it difficult for current CLSO image alignment techniques to provide good alignment since the NICC energy functional is highly dependent on linear intensity homogeneity among all the images.

The main contribution of this paper is a robust approach to CLSO photoreceptor image alignment under differing polarization states using a novel energy functional based on complex phase relationships. The proposed method is invariant to the effects of polarization and scale, robust to noise, and highly sensitive to photoreceptor structural characteristics, which are the key challenges to CLSO photoreceptor image alignment under differing polarization states. In this paper, the materials and methods are described in Section II, and the experimental results using in vivo fish cone photoreceptor images acquired under different polarization states are presented in Section III.

\section{MATERIALS AND METHODS}

The polarimetric CSLO enhancement system used in this paper is the same as that described by Bueno et al. [8]. An HeNe laser beam is passed through a polarization state generator unit composed of a fixed vertical linear polarizer and a rotating quarter-wave plate (QWP) to achieve the desired polarization state. The beam from the generator unit is then scanned by an $X-Y$ scanning unit before reaching the eye and focused on a small portion of the retina to image photoreceptors. The reflected light from the eye is descanned and passed through a collector lens and a confocal pinhole. Finally, the reflected light is amplified by a photomultiplier tube before being recorded by a computer. At a given location, video sequences are recorded for four independent incoming polarization states by rotating the QWP. Based on the four CLSO photoreceptor images recorded at different polarization states, the spatially resolved elements of the first row of the Mueller matrix of the combined eye and instrument can be computed. These elements are sufficient for constructing the photoreceptor image for any incoming polarized state [8]. By constructing a large number of Mueller images and selecting the highest quality images using image quality metrics, better visualization of the fundus structures can be achieved. To reduce the effect of misalignments due to eye movements during the recording process on the computation of the Mueller matrix, the corresponding images acquired from the four different polarization states must first be aligned to each other. As such, the alignment stage is vital for proper Mueller matrix computation as well as the quality of the constructed Mueller images.

\section{A. Energy Functional Based on Complex Phase Relationships}

An important factor that affects the performance of an automated alignment method is the underlying energy functional, which expresses the dissimilarity between two images. The optimal alignment is determined by minimizing the energy functional. Hence, it is imperative that the energy functional used be designed to address the issues associated with aligning CLSO photoreceptor images, such as low SNR, poor structural contrast, and intensity inhomogeneities associated with differing polarization states. In current methods used to align retinal images acquired using ophthalmoscopic imaging [5], [8], [9], the energy functional $E$ is defined as the negative NICC between two images. However, this energy functional is ill-suited for the alignment of CLSO photoreceptor images for several reasons. First, the NICC energy functional assumes a strict linear relationship between the corresponding image intensities, which is typically not the case due to intensity inhomogeneities exhibited by CLSO images acquired under differing polarization states. Second, the NICC energy functional relies on the image intensities to measure the dissimilarity between two images. However, CLSO photoreceptor images are characterized by poor structural contrast [5], thus making the use of image intensities a poor choice for measuring the dissimilarity between photoreceptor structures. Finally, the NICC energy functional is highly sensitive to outlier noise [10], thus resulting in poor alignment performance for images characterized by low SNR. Therefore, an alternative energy functional that addresses all of the aforementioned issues is highly desired.

One useful approach to designing the energy functional is to address each of the aforementioned challenges in a systematic fashion. The first challenge that needs to be addressed in the design of the energy functional is in dealing with the intensity differences and inhomogeneities inherent in CLSO photoreceptor images due to the complex polarization properties of fundus structures. These polarization-related intensity variations makes it difficult to compare images based on their intensity values, as is done in NICC energy functionals used by existing CLSO image alignment techniques, since the same photoreceptor structural characteristics within the images may be represented by different intensity values under different polarization states. One approach to dealing with such polarization-related intensity variation issues is to construct an alternative image representation that is invariant to polarization. As the basis of our energy functional, we propose a new polarization-invariant image representation based on structural characteristics. This is motivated by the fact that two CLSO images acquired from the same photoreceptors under different polarization states share similar structural characteristics even if the underlying intensity 
characteristics are very different. To capture the structural characteristics of the photoreceptors in a way that is invariant to polarization, we propose the use of complex phase relationships, which has been shown in recent studies to be very effective at representing structural characteristics in natural scenes [11]-[16]. By relying only on complex phase information, the proposed image representation is invariant to polarizationrelated image inhomogeneities.

One approach to constructing a complex phase-based image representation can be described as follows. Given an $i \times j$ image $f$, an overcomplete log-Gabor complex wavelet transform [17] is used to decompose $f$ into a multiscale decomposition, where the dimension of each scale $(s=1, \ldots, m)$ is $i \times j$. At each point $\underline{x}=(x, y)$ of each scale $s$, there exists a set of $\varsigma$ complex coefficients $\wp=$ $\left\{A_{s}\left(\underline{x}, \theta_{1}\right) e^{j \phi_{s}\left(\underline{x}, \theta_{1}\right)}, \ldots, A_{s}\left(\underline{x}, \theta_{\varsigma}\right) e^{j \phi_{s}\left(\underline{x}, \theta_{\varsigma}\right)}\right\}$, which corresponds to the wavelet responses to the corresponding $\varsigma$ orientations. The amplitude $A_{s}(\underline{x}, \theta)$ and phase $\phi_{s}(\underline{x}, \theta)$ components of each complex coefficient can be expressed as

$$
A_{s}(\underline{x}, \theta)=\sqrt{\left(f(\underline{x}) * R_{s}^{e}(\theta)\right)^{2}+\left(f(\underline{x}) * R_{s}^{o}(\theta)\right)^{2}}
$$

and

$$
\phi_{s}(\underline{x}, \theta)=\tan ^{-1}\left(\frac{\left(f(\underline{x}) * R_{s}^{e}(\theta)\right)}{\left(f(\underline{x}) * R_{s}^{o}(\theta)\right)}\right)
$$

where $R_{s}^{e}(\theta)$ and $R_{s}^{o}(\theta)$ corresponds to the even- and oddsymmetric log-Gabor quadrature pair at scale $s$ and orientation $\theta$. To capture the structural characteristics of natural images, Morrone and Owens [11] proposed that the structural significance at a point $\underline{x}$ can be quantified as the normalized weighted summation of cosine-weighted complex phase deviations from the mean phase $\bar{\phi}(\underline{x}, \theta)$ across $m$ scales and $\varsigma$ orientations

$$
\varrho(\underline{x})=\frac{\sum_{q=1}^{\varsigma} \sum_{s=1}^{m} A_{s}\left(\underline{x}, \theta_{q}\right) \cos \left(\phi_{s}\left(\underline{x}, \theta_{q}\right)-\bar{\phi}\left(\underline{x}, \theta_{q}\right)\right)}{\sum_{q=1}^{\varsigma} \sum_{s=1}^{m} A_{s}\left(\underline{x}, \theta_{q}\right)} .
$$

The motivation for the measure introduced by Morrone and Owens [11] is the theory that the high phase order corresponds to significant structural characteristics in images. It can be seen from (3) that as phase order across scales and orientations increases, corresponding to increasing structural significance, the cosine-weighted complex phase deviations approach one and $\varrho(\underline{x})$ approaches one as well. Conversely, as phase order decreases, corresponding to decreasing structural significance, the cosine-weighted complex phase deviations approach negative one and $\varrho(\underline{x})$ approaches negative one as well. As such, it can be seen that complex phase relationships can be very effective at providing a polarization-invariant image representation of the photoreceptors, thus addressing the issue associated with polarization-related intensity differences and inhomogeneities. Furthermore, by utilizing phase relationships across multiple scales, the resulting image representation is also invariant to scale. Therefore, given these benefits, the concept of complex phase relationships will act as the basis of image representation in the proposed energy functional.

The second challenge that needs to be addressed in the design of the energy functional is in dealing with the poor structural contrast inherent in CLSO photoreceptor images due to both the underlying photoreceptor structures as well as the mechanisms of the imaging system [5]. Poor structural contrast makes it difficult to capture and distinguish fine structural detail pertaining to the photoreceptors, which is very important in quantifying the structural dissimilarities between two images. One approach to addressing the issues associated with poor structural contrast is to increase the response sensitivity of the energy functional to the photoreceptor structural characteristics. Since the proposed energy functional relies on complex phase relationships to capture and distinguish photoreceptor structural characteristics, it is important to first study the behavior of the measure $\rho(\underline{x})$ described in (3) with respect to phase order. Let $\varpi$ represent the phase deviation between a phase angle $\phi(\underline{x}, \theta)$ and the mean phase angle $\bar{\phi}(\underline{x}, \theta)$

$$
\varpi=\phi_{s}(\underline{x}, \theta)-\bar{\phi}(\underline{x}, \theta) .
$$

It can be observed in (3) that $\rho(\underline{x})$ is proportional to a periodic phase deviation weighing function $\Lambda=\cos (\varpi)$. As such, we can simplify the analysis by studying the behavior of $\Lambda$. It can also be observed in (3) that the absolute value of the phase deviation term $|\varpi|$ decreases as phase order increases, which corresponds to an increase in structural significance. Furthermore, given the properties of the cosine function, the decrease of $|\varpi|$ results in an increase of $\Lambda$. Therefore, one can study the response sensitivity of $\Lambda$ to phase order by taking the derivative of $\Lambda$ with respect to $\varpi$

$$
\frac{\partial \Lambda}{\partial \varpi}=-\sin (\varpi)
$$

A plot of both $\Lambda=\cos (\varpi)$ and its derivative $\partial \Lambda / \partial \varpi$ is shown in Fig. 1(a). It can be observed from $\partial \Lambda / \partial \varpi$ that the rate of change of $\Lambda$ near the peak of $\Lambda$ (where $\varpi$ is minimal and phase order is maximal) is very slow and gradual. For example, a reasonably large phase deviation of $37^{\circ}$ would still result in a large value of $\Lambda$ that is $80 \%$ of the maximum value of $\Lambda$. This slow, gradual rate of change near the peak of $\Lambda$ results in the poor response sensitivity of $\rho(\underline{x})$ to phase order, and consequently to significant structural characteristics.

In an attempt to address this issue, Kovesi [15] proposed an alternative periodic phase deviation weighing function that exhibits a sharper rate of change near the peak of $\Lambda$, as shown in Fig. 1(b). While this weighing function increases the response sensitivity of $\rho(\underline{x})$ to significant structural characteristics when compared to the cosine weighing function proposed by Morrone and Owens [11], there are two important limitations that need to be considered. First, the weighing function decreases almost linearly from minimum $|\varpi|$ to maximum $|\varpi|$. As such, the response sensitivity of the weighing function to phase order and consequently to significant structural characteristics does not noticeably increase as phase order increases. Hence, the weighing function does not emphasize structural characteristics with high structural significance, which is important in capturing and distinguishing fine structural detail pertaining to the photoreceptors. More importantly, the weighing function exhibits regions where the decrease of $|\varpi|$ actually results in a decrease of $\Lambda$, which is counterintuitive. To address all of the aforementioned 

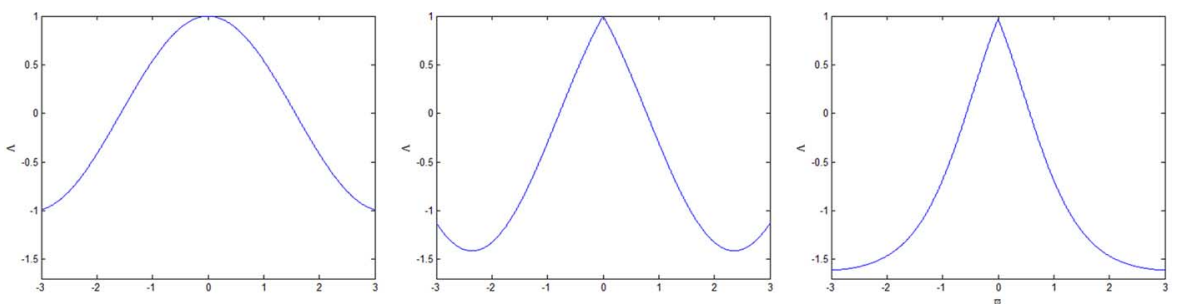

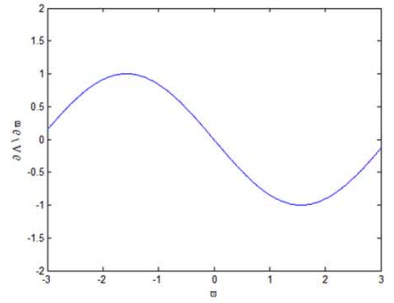

(a)

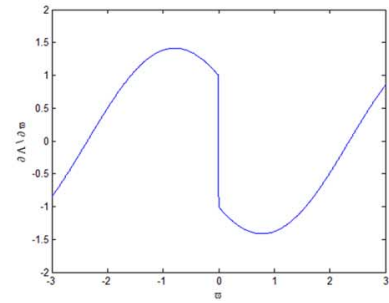

(b)

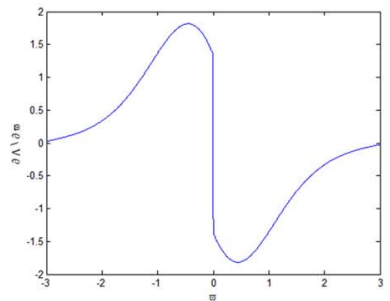

(c)

Fig. 1. Plots of $\Lambda$ and its derivative $\partial \Lambda / \partial \varpi$. (a) Weighing function proposed by Morrone and Owens [11]. (b) Weighing function proposed by Kovesi [15]. (c) Proposed weighing function. It can be observed that the response sensitivity of the proposed weighing function to significant structural characteristics is noticeably higher than the other weighing functions. It can also be observed that the proposed weighing function does not suffer from the undesirable phenomenon exhibited by the weighing function proposed by Kovesi [15], where the function $\Lambda$ decreases as phase deviation $|\varpi|$ decreases in certain regions.

limitations associated with the weighing function proposed by Kovesi [15], we propose an alternative periodic weighing function based on the exponential difference of sinusoids

$$
\Lambda_{\nu}=e^{\cos (\varpi)-|\sin (\varpi / 2)|}-\frac{3}{2} .
$$

The corresponding derivative $\partial \Lambda_{\nu} / \partial \varpi$ can be expressed as

$$
\begin{aligned}
& \frac{\partial \Lambda_{\nu}}{\partial \varpi}=e^{\cos (\varpi)-|\sin (\varpi / 2)|} \\
& \quad \times\left(-\sin (\varpi)-\frac{1}{2} \frac{\partial|\sin (\varpi / 2)|}{\partial \varpi} \cos \left(\frac{\varpi}{2}\right)\right) .
\end{aligned}
$$

The plots of the proposed weighing function and its corresponding derivative are shown in Fig. 1(c). It can be observed that the proposed weighing function emphasizes high phase order (low $|\varpi|)$, as indicated by the high rate of change near the peak of $\Lambda_{\nu}$. Furthermore, it can be observed that the proposed weighing function $\Lambda$ never decreases as phase deviation $|\varpi|$ decreases, thus avoiding the undesirable phenomenon exhibited by the weighing function proposed by Kovesi [15]. Given this new weighing function $\Lambda_{\nu}$, the final complex phase-based image representation $\varrho_{\nu}(\underline{x})$ used by the proposed energy functional can be expressed as shown in (8), at the bottom of the next page, where $\xi$ is a small constant to handle situations where all the amplitude components are zero.

The third and final challenge that needs to be addressed in the design of the energy functional is in dealing with the low SNR that characterizes CLSO retinal photoreceptor images due to the nature of the imaging system [5]. This is partially addressed in $\varrho_{\nu}(\underline{x})$, which deemphasizes structural characteristics with low phase order such as noise. As mentioned earlier, the NICC energy functional is highly sensitive to outlier noise [10]. This is problematic given the fact that CLSO images are characterized by low contrast resolution and low SNR that can result in outliers. To reduce the effect of outlier noise on alignment accuracy, we propose the use of a Pearson type VII distance metric [18] as the basis for quantifying the dissimilarity between CLSO photoreceptor images in the proposed energy functional

$$
\psi(u, v)=\ln \left(\sqrt{1+(u-v)^{2}}\right) .
$$

To analyze the influence of outlier noise on (9), one approach is to study its derivative with respect to $\varepsilon=u-v$ [21]

$$
\frac{\partial \psi}{\partial \varepsilon}=\frac{\varepsilon}{\left(1+\varepsilon^{2}\right)} .
$$

It can be observed from (10) that the Pearson type VII distance metric is a redescending error metric since the influence of outlier noise, which is proportional to $\partial \psi / \partial \varepsilon$, is bounded and tends to zero. Therefore, given two CLSO photoreceptor images $f(\underline{x})$ and $g(\underline{x})$, the proposed energy functional $E(f, g)$ can be expressed as the cumulative Pearson type VII distance between the complex phase-based image representations $\varrho_{\nu, f}(\underline{x})$ and $\varrho_{\nu, g}(\underline{x})$ of $f(\underline{x})$ and $g(\underline{x})$, respectively

$$
E(f, g)=\sum_{\underline{x}} \ln \left(\sqrt{1+\left(\rho_{\nu, f}(\underline{x})-\rho_{\nu, g}(\underline{x})\right)^{2}}\right) .
$$

Finally, to further reduce the effect of noise without affecting the structural detail of the photoreceptors, we utilize the adaptive bilateral estimation scheme proposed by Wong [19], which has been shown to provide good noise removal while preserving structural detail, to estimate the original noise-free CLSO image prior to computing the energy functional.

\section{B. Constrained Optimization Using Sequential Quadratic Programming}

Based on the energy functional proposed in Section II-A, the CLSO photoreceptor alignment problem can be formulated as an optimization problem, where the underlying goal is to estimate 
the transformation $\hat{T}$ between images $f$ and $g$ that minimizes the energy functional $E$

$$
\hat{T}=\underset{T}{\arg \min }[E(f, T(g))] .
$$

It was shown that the transformation between CLSO images can be well modeled by $x$ - and $y$-translation $\underline{\kappa}=\left\{\kappa_{x}, \kappa_{y}\right\}$ and rotation $\vartheta$ [5], [8]. Furthermore, to reduce computational time, the transform range for each of the transform parameters was limited to realistic values. Based on this information, the final CLSO photoreceptor alignment problem can be formulated as the following constrained optimization problem:

$$
\{\underline{\hat{\hat{k}}}, \hat{\vartheta}\}=\underset{\{\underline{\kappa}, \vartheta\}}{\arg \min }\left[\sum_{\underline{x}} \ln \left(\sqrt{1+\left(\rho_{\nu, f}(\underline{x})-\rho_{\nu, \Upsilon_{\vartheta} g+\underline{\kappa}}(\underline{x})\right)^{2}}\right)\right]
$$

where $\Upsilon_{\vartheta}$ is the rotation matrix for $\vartheta$, and subject to

$$
\begin{aligned}
& -\underline{\alpha} \leq \underline{\kappa} \leq \underline{\alpha} \\
& -\beta \leq \vartheta \leq \beta .
\end{aligned}
$$

For test purposes, $\underline{\alpha}=(15,15)$ pixels and $\vartheta=2^{\circ}$, which are sufficient for real-world scenarios. To solve this constrained optimization problem, a sequential quadratic programming (SQP) approach [20] was employed. In the proposed method, the transformation parameters $\left\{\Theta_{1}, \Theta_{2}, \Theta_{3}\right\}=\left\{\underline{\kappa}_{x}, \underline{\kappa}_{y}, \vartheta\right\}$ are estimated iteratively based on the energy functional $E$

$$
\hat{\Theta}_{i}^{k+1}=\hat{\Theta}_{i}^{k}+\gamma_{i}^{k} d_{i}^{k}
$$

where $k$ denotes an iteration, $\gamma_{i}$ is a nonnegative step size, and $d_{i}$ is the step direction calculated by solving a quadratic subprogram involving the energy functional $E$ with linear approximations of the constraint functions [20]. Based on the estimated parameters, $g$ is transformed and the energy functional $E$ is recomputed between $f$ and the transformed $g$. This process is repeated until convergence is achieved to obtain the optimal alignment between $f$ and $g$.

\section{EXPERIMENTAL RESULTS}

To evaluate the effectiveness of the proposed method for the purpose of aligning CLSO photoreceptor images, the proposed method was implemented and evaluated using real CSLO fish cone photoreceptor images acquired using the experimental setup described in Section II. The CLSO images were acquired under four different polarization states by rotating the fast axis of the QWP to orientations of $-45^{\circ}, 0^{\circ}, 30^{\circ}$, and $60^{\circ}$. Examples of these CLSO photoreceptor images are shown in Fig. 2. Based on the acquired CLSO images at different polarization states, three image test pairs were constructed as follows.

1) $P A$ : Image pair with QWP orientations of $0^{\circ}$ and $-45^{\circ}$.

2) $P B$ : Image pair with QWP orientations of $0^{\circ}$ and $30^{\circ}$.

3) $P C$ : Image pair with QWP orientations of $0^{\circ}$ and $60^{\circ}$.
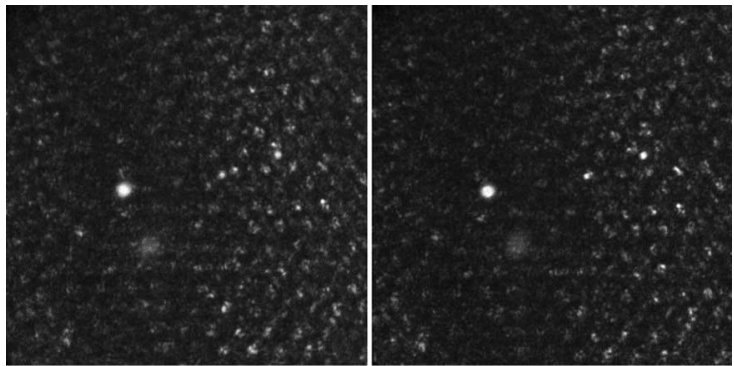

(a)

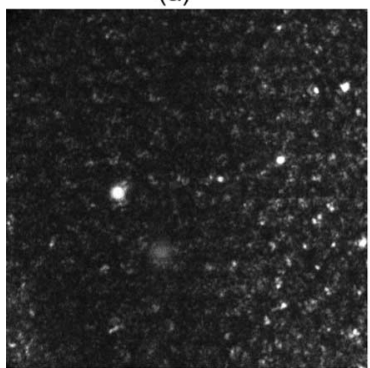

(c)

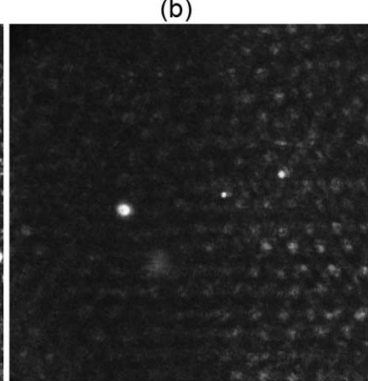

(d)
Fig. 2. Examples of CLSO photoreceptor images acquired with QWP orientations. (a) $-45^{\circ}$. (b) $0^{\circ}$. (c) $30^{\circ}$. (d) $60^{\circ}$. Note that the large, consistent bright spots are a result of lens reflectance and are masked out automatically as the fixed location of these bright spots are known.

Each of the image test pairs was manually aligned by a human expert to establish the gold-standard transformation between the CLSO photoreceptor images for evaluation purposes.

\section{A. Experiment 1}

The first set of experiments investigates the behavior of the proposed energy functional and the NICC energy functionals used by current ophthalmoscopic imaging alignment techniques [5], [8], [9]. In particular, the monotonicity and the location of the global minima of the energy functional with respect to transformation play a crucial role in the overall alignment accuracy of an automated alignment method. The monotonicity of an energy functional with respect to transformation is important as it affects the ability for iterative optimization methods such as the SQP approach to converge to the global minima. In situations where the energy functional exhibits highly nonmonotonic behavior with respect to transformation, iterative optimization methods have a higher probability of converging to a local minima as opposed to the global minima. The location of the global minima of an energy functional with respect to transformation is also very important as it affects the ability for the alignment method to achieve the correct alignment between the images. In situations where the global minima of the energy functional is located away from the correct alignment, iterative optimization methods will converge to a solution that does not yield the proper alignment between the images. The first set of experiments is performed by evaluating the proposed energy functional and the

$$
\varrho_{\nu}(\underline{x})=\frac{\sum_{q=1}^{\varsigma} \sum_{s=1}^{m} A_{s}\left(\underline{x}, \theta_{q}\right)\left(\exp \left[\cos \left(\phi_{s}\left(\underline{x}, \theta_{q}\right)-\bar{\phi}\left(\underline{x}, \theta_{q}\right)\right)-\left|\sin \left(\left[\phi_{s}\left(\underline{x}, \theta_{q}\right)-\bar{\phi}\left(\underline{x}, \theta_{q}\right)\right] / 2\right)\right|\right]-(3 / 2)\right)}{\sum_{q=1}^{\varsigma} \sum_{s=1}^{m} A_{s}\left(\underline{x}, \theta_{q}\right)+\xi}
$$



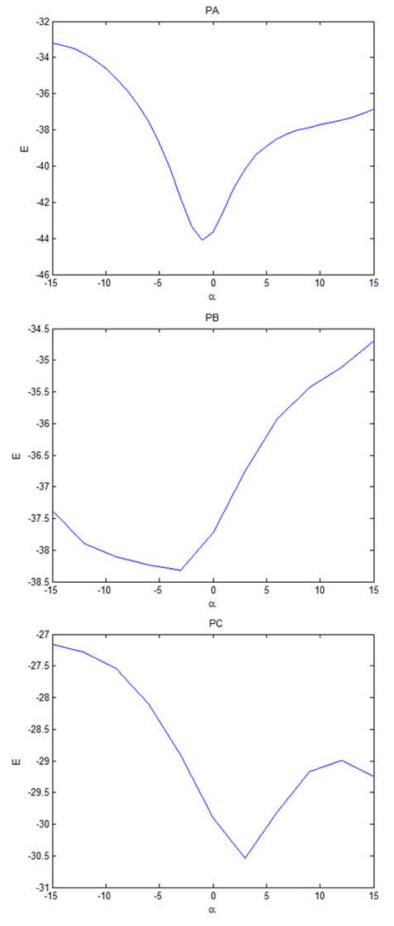

(a)
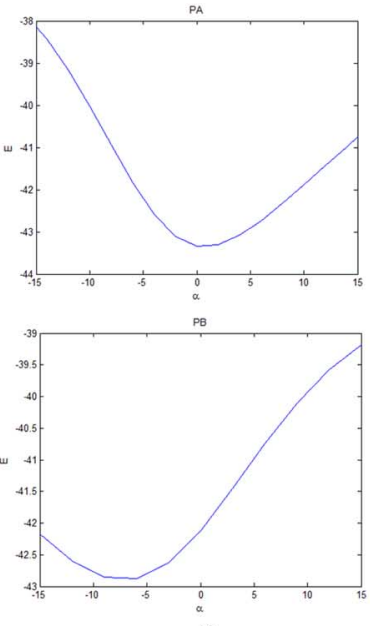

$\mathrm{PC}$

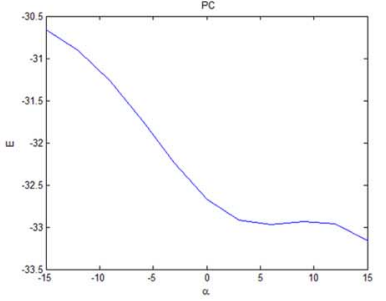

(b)
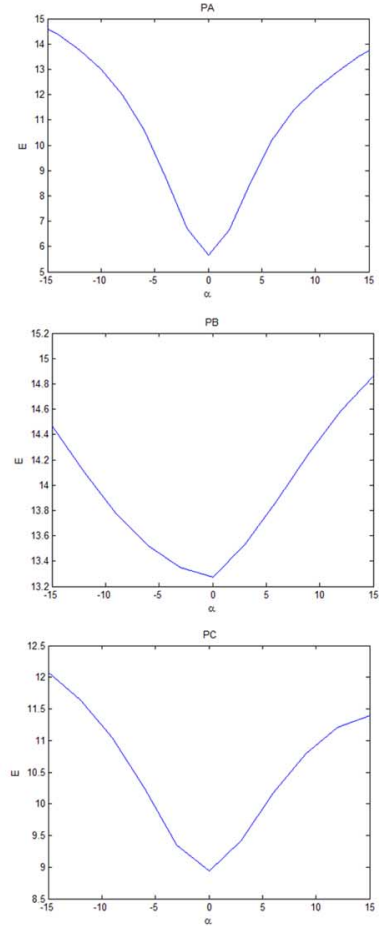

(c)

Fig. 3. Energy functional with respect to translation for three image test pairs. (a) NICCA [8], [9]. (b) NICCB [5]. (c) Proposed energy functional.

NICC energy functionals as described by Goatman et al. [9] (which is also used by Bueno et al. [8]) (NICCA) and by Wade and Fitzke [5] (NICCB) over horizontal translations within the range of $[-15,15]$ pixels, as specified in $\underline{\alpha}$. This experiment was performed on all three image test pairs.

The energy functionals with respect to translation are shown in Fig. 3. It can be observed that there are significant offsets between the global minimum of the energy functional proposed in [8] and [9] and the gold-standard alignment for all of the image test pairs. It can also be observed that there are noticeable global minimum offsets for the energy functional proposed in [5] for all but the test pair PA. This is not the case for the proposed energy functional, where the global minimum of the energy functional coincides with the gold-standard alignment for all of the image test pairs. Furthermore, it can be observed that, for test pair PC, the energy functions proposed [5], [8], and [9] both exhibit local minima. This is not the case for the proposed energy functional, which remains monotonic. These results demonstrate the effectiveness of the proposed energy functional in scenarios characterized by low SNR, poor contrast, and polarization-related image inhomogeneities.

\section{B. Experiment 2}

The second set of experiments investigates the alignment accuracy of the proposed method with the methods proposed in [5], [8], and [9] (denoted as NICCA and NICCB, respectively) using all three image test pairs. Each image test pair was misaligned using 20 random transformations involving translation and rotation within the range of $[-\alpha, \alpha]$ and $[-\beta, \beta]$, respectively, resulting in a total of 60 test cases. To evaluate
TABLE I

ALIGNMENT ACCURACY FOR EXPERIMENT 2

\begin{tabular}{|c|c|c|c|}
\hline \hline \multirow{2}{*}{$\begin{array}{c}\text { Test } \\
\text { Pair }\end{array}$} & \multicolumn{3}{|c|}{ Alignment Error ${ }^{1}\left(\epsilon_{\theta}, \epsilon_{x}, \epsilon_{y}\right)$} \\
\cline { 2 - 4 } & NICCA [9], [8] & NICCB [5] & Proposed Method \\
\hline \hline \multicolumn{3}{|c|}{ min } \\
\hline \hline PA & $\left(0.293^{\circ}, 1.178,1.923\right)$ & $\left(0.234^{\circ}, 0.453,0.389\right)$ & $\left(0.105^{\circ}, 0.361,0.459\right)$ \\
PB & $\left(0.396^{\circ}, 2.941,3.112\right)$ & $\left(0.446^{\circ}, 6.907,7.639\right)$ & $\left(0.189^{\circ}, 0.268,0.180\right)$ \\
PC & $\left(0.149^{\circ}, 3.170,2.639\right)$ & $\left(0.299^{\circ}, 5.466,6.948\right)$ & $\left(0.117^{\circ}, 0.115,0.165\right)$ \\
\hline \hline \multicolumn{4}{|c|}{ mean } \\
\hline \hline PA & $\left(0.603^{\circ}, 1.992,2.258\right)$ & $\left(0.510^{\circ}, 0.693,0.803\right)$ & $\left(0.367^{\circ}, 0.508,0.680\right)$ \\
PB & $\left(0.561^{\circ}, 3.163,3.969\right)$ & $\left(1.027^{\circ}, 9.284,8.629\right)$ & $\left(0.233^{\circ}, 0.382,0.415\right)$ \\
PC & $\left(0.631^{\circ}, 8.114,7.373\right)$ & $\left(0.919^{\circ}, 8.797,8.245\right)$ & $\left(0.484^{\circ}, 0.388,0.315\right)$ \\
\hline \hline \multicolumn{4}{|c|}{ max } \\
\hline \hline PA & $\left(1.017^{\circ}, 2.106,2.389\right)$ & $\left(1.22^{\circ}, 1.149,1.805\right)$ & $\left(0.607^{\circ}, 0.607,0.724\right)$ \\
PB & $\left(0.866^{\circ}, 3.769,4.246\right)$ & $\left(1.53^{\circ}, 14.10,13.89\right)$ & $\left(0.624^{\circ}, 0.857,0.735\right)$ \\
PC & $\left(0.984^{\circ}, 15.00,13.28\right)$ & $\left(1.32^{\circ}, 15.00,14.81\right)$ & $\left(0.850^{\circ}, 0.553,0.637\right)$ \\
\hline \hline
\end{tabular}

${ }^{1}$ The table reports the minimum/mean/maximum of the 20 trials.

the alignment accuracy of the methods under test, each of the methods was applied to align all 60 test cases, and the alignment error $\epsilon_{\theta}, \epsilon_{x}, \epsilon_{y}$ for each parameter was computed.

A summary of the alignment accuracy for the second set of experiments is shown in Table I. It can be observed that the mean alignment error $\epsilon_{\theta}, \epsilon_{x}, \epsilon_{y}$ was noticeably lower for the proposed method when compared to the methods proposed in [5], [8], and [9] for all of the image test pairs. The difference images for a sample test case for the test pair PA before and after alignment using the proposed method are shown in Fig. 4. It can be seen that the proposed method achieved good image alignment results, as the difference image for the test image pair after alignment is minimized compared to the difference image before alignment. The remaining differences in the difference image after alignment are due to polarization-related 

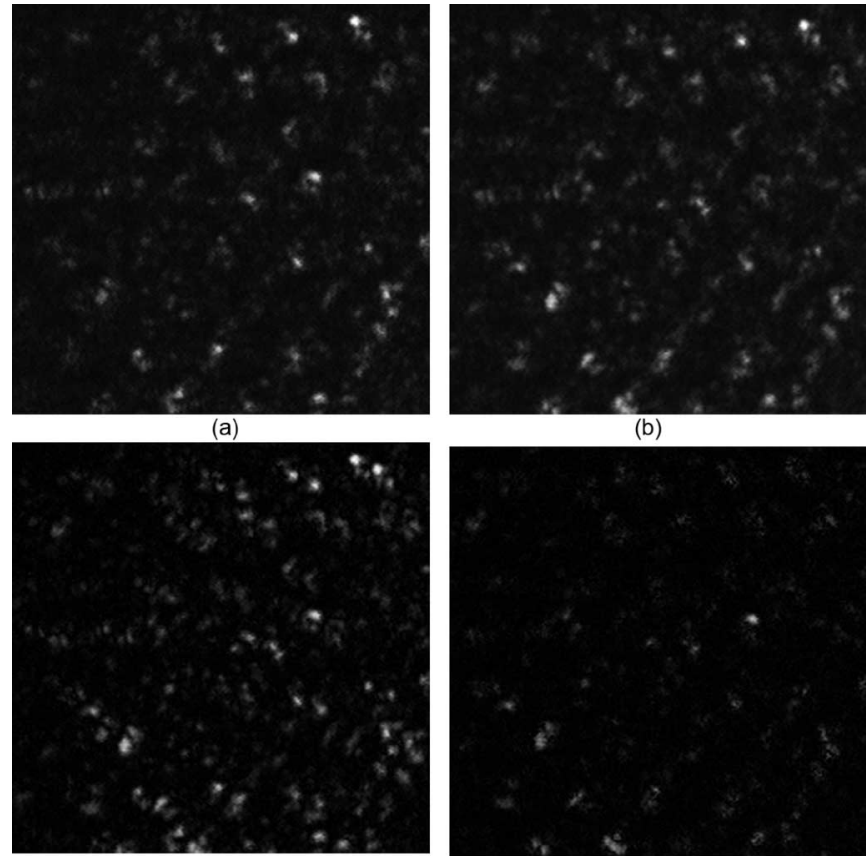

(c)

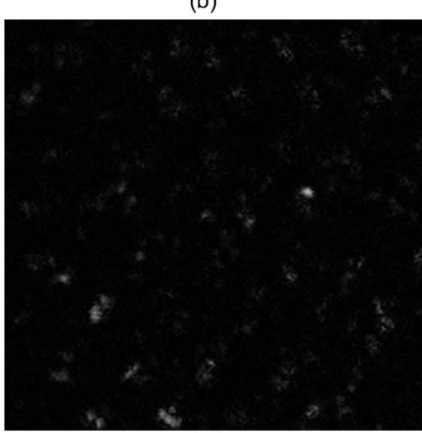

(d)

Fig. 4. Sample alignment result for test pair PA (zoomed in for clarify). (a) QWP orientation of $0^{\circ}$. (b) QWP orientations of $-45^{\circ}$. (c) Difference image before alignment. (d) Difference image after alignment using the proposed method. It can be seen that the images were successfully aligned.

intensity differences as opposed to due to misalignment. These results demonstrate the effectiveness of the proposed method for aligning CLSO photoreceptor images acquired under different polarization states.

\section{CONCLUSION}

In this paper, we introduced a novel automated approach to aligning CLSO photoreceptor images acquired under differing polarization states using complex phase relationships. A novel invariant energy functional was introduced that addresses important issues such as low SNRs, poor contrast, and polarizationrelated image inhomogeneities. An SQP approach was described for determining the optimal alignment between the CLSO photoreceptor images. Experiments using CLSO fish cone photoreceptor images demonstrate that good alignment accuracy can be achieved. Future work involves investigating alternate optimization and noise suppression methods, as well as alternative energy functionals for improving alignment accuracy.

\section{ACKNOWLEDGMENT}

The author would like to thank Dr. M. Campbell from the School of Optometry, University of Waterloo for providing the CLSO retinal photoreceptor images used during testing.

\section{REFERENCES}

[1] G. Fishman, "Progressive human cone-rod dysfunction (dystrophy)," Trans. Amer. Acad. Ophthalmol. Otolaryngol., vol. 81, pp. 716-724, 1976.

[2] S. Choi, N. Doble, J. Hardy, S. Jones, J. Keltner, S. Olivier, and J. Werner, "In vivo imaging of the photoreceptor mosaic in retinal dystrophies and correlations with visual function," Invest. Ophthalmol. Vis. Sci., vol. 47 no. 5, pp. 2080-2092, 2006.

[3] G. Farrar, P. Kenna, and P. Humphries, "On the genetics of retinitis pigmentosa and on mutation-independent approaches to therapeutic intervention," EMBO J., vol. 21, no. 5, pp. 857-864, 2002.

[4] A. Manivannan, P. Sharp, R. Phillips, and J. Forrester, "Digital fundus imaging using a scanning laser ophthalmoscope," Physiol. Meas., vol. 14, pp. 43-56, 1993.

[5] A. Wade and F. Fitzke, "A fast, robust pattern recognition asystem for low light level image registration and its application to retinal imaging," Opt. Exp., vol. 3, no. 5, pp. 190-119, 1998.

[6] J. Bueno and M. Campbell, "Confocal scanning laser ophthalmoscopy improvement by use of Mueller-matrix polarimetry," Opt. Lett., vol. 27, pp. 830-832, 2002.

[7] Y. Zhang, S. Poonja, and A. Roorda, "MEMS-based adaptive optics scanning laser ophthalmoscopy," Opt. Lett., vol. 31, no. 9, pp. 1268-1270, 2006.

[8] J. Bueno, J. Hunter, C. Cookson, M. Kisilak, and M. Campbell, "Improved scanning laser fundus imaging using polarimetry," J. Opt. Soc. Amer. A. Opt. Image. Sci. Vis., vol. 24, pp. 1337-1348, 2007.

[9] K. Goatman, A. Manivannan, J. Hipwell, P. Sharp, N. Lois, and J. Forrester, "Automatic registration and averaging of ophthalmic autofluorescence images," presented at the Med. Image Understanding Anal. (MIUA), Univ. Birmingham, Birmingham, U.K., 2001.

[10] J. Kim and J. Fessler, "Intensity-based image registration using robust correlation coefficients," IEEE Trans. Med. Imag., vol. 23, no. 11, pp. 14301444, Nov. 2004

[11] M. Morrone and R. Owens, "Feature detection from local energy," Pattern Recognit. Lett., vol. 6, pp. 303-313, 1987.

[12] M. Morrone and D. Burr, "Feature detection in human vision: A phasedependent energy model," Proc. R. Soc. Lond. B, vol. 235, pp. 221-245, 1988.

[13] A. V. Oppenheim and J. S. Lim, "The importance of phase in signals," Proc. IEEE, vol. 69, no. 5, pp. 529-541, May 1981.

[14] M. Thomson, "Visual coding and the phase structure of natural scenes," Netw: Comput. Neural Syst., vol. 10, pp. 123-132, 1999.

[15] P. Kovesi, "Phase congruency: A low-level image invariant," Psychol. Res., vol. 64, no. 2, pp. 136-148, 2000.

[16] Z. Wang and E. Simoncelli, "Local phase coherence and the perception of blur," Adv. Neural Inf. Process. Syst., vol. 16, pp. 786-792, May 2004.

[17] D. Field, "Relations between the statistics of natural images and the response properties of cortical cells," J. Opt. Soc. Amer. A, vol. 4, no. 12, pp. 2379-2394, 1987.

[18] K. Pearson, "Mathematical contributions to the theory of evolution, XIX: Second supplement to a memoir on skew variation," Philos. Trans. R. Soc. Lond. Ser. A, Containing Papers Math. Phys. Character, vol. 216, pp. 429-457, 1916.

[19] A. Wong, "Adaptive bilateral filtering of image signals using local phase characteristics," Signal Process., vol. 88, no. 6, pp. 1615-1619, 2008.

[20] P. Boggs and J. Tolle, "Sequential quadratic programming," Acta Numer. vol. 4, pp. 1-51, 1995.

[21] M. Black and A. Rangarajan, "The outlier process: Unifying line processes and robust statistics," in Proc. IEEE CVPR, 1994, pp. 15-22.

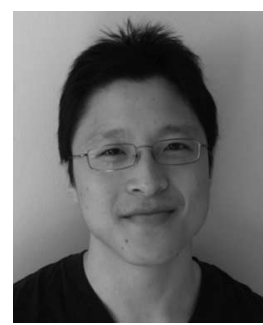

Alexander Wong (M'05) received the B.Sc. degree in computer engineering and the M.Sc. degree in electrical and computer engineering in 2005 and 2007, respectively, from the University of Waterloo, Waterloo, ON, Canada, where he is currently working toward the Ph.D. degree in the Department of Systems Design Engineering. He is also with the Vision and Image Processing Research Group, University of Waterloo. He has authored or coauthored papers in various fields such as computer vision, graphics, image processing, biomedical signal processing, and multimedia systems, published in refereed journals and conferences. His current research interests include biomedical image processing and analysis, computer vision, and pattern recognition. He has was involved in the projects on image registration, image denoising, image superresolution, image segmentation, biomedical tracking, biomedical image analysis, and image and video coding. 